\title{
O impacto das restrições financeiras no investimento e nas reservas de caixa das PME do setor da metalomecânica
}

\section{The impact of financial constraints on the investment and cash holdings of metalworking sector SMEs}

\author{
Marília Veiga \\ Instituto Superior de Contabilidade e Administração do Porto, Portugal \\ mariliaveiga@sapo.pt \\ Sónia Silva \\ Escola Economia e Gestão, Universidade do Minho, Portugal \\ sonia@eeg.uminho.pt
}

\begin{abstract}
Resumo
O objetivo principal deste trabalho é analisar o impacto das restrições financeiras no investimento e nas reservas de caixa das pequenas e médias empresas portuguesas do setor da metalomecânica. A informação foi recolhida da base de dados SABI, entre 2006 e 2016. Dada a estrutura longitudinal dos nossos dados, as hipóteses foram testadas com recurso a metodologias de dados em painel, nomeadamente através de modelos de efeitos fixos. Os nossos resultados sugerem que as empresas que enfrentam maiores restrições financeiras revelam maior sensibilidade do investimento ao financiamento interno e mantêm maiores reservas de caixa em relação ao cash flow. Por outro lado, as empresas exportadoras aparentam ter maior sensibilidade do seu investimento e das suas reservas de caixa ao cash flow do que as empresas domésticas. Também as empresas que exportam para dentro e para fora do Mercado Comunitário apresentam maior sensibilidade do investimento e das reservas de caixa ao cash flow.
\end{abstract}

Palavras-chave: restrições financeiras; investimento; reservas de caixa; cash flow.

\begin{abstract}
The main objective of this work is to analyze the impact of financial constraints on the investment and cash holdings of small and medium-sized Portuguese companies in the metalworking sector. The data was collected from the SABI database, from 2006 to 2016. Given the longitudinal structure of our data, the hypotheses were tested using panel data methodologies, namely by fixed effects models. Our results suggest that firms facing more financial constraints reveal greater investment sensitivity to the internal financing and save more money in relation to cash flows. On the other hand, our results suggest that exporters display higher investment and cash sensitivities to the cash flow than domestic firms. In addition, companies that export inside and outside the EU market appear to have greater sensitivity to their investment and cash holdings to the cash flow.
\end{abstract}

Keywords: financial constraints; investment; cash holdings; cash flow.

Dos Algarves: A Multidisciplinary e-Journal, 35 - 2019.

ISBN 2182-5580 @ ESGHT - University of the Algarve, Portugal.

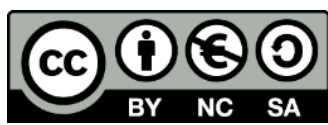

To cite this article: Veiga, M. \& Silva, S. (2019). O impacto das restrições financeiras no investimento e nas reservas de caixa das PME do setor da metalomecânica. Dos Algarves: A Multidisciplinary e-Journal, 35, 1-19. DOI: 10.18089/DAMeJ.2019.35.1 


\section{Introdução}

Fazzari, Hubbard e Petersen (1988) formularam a hipótese de que, perante restrições financeiras, o investimento depende, sobretudo, do financiamento interno, isto é, do cash flow; esta hipótese está longe de ser consensual na literatura das restrições financeiras. No entanto, apesar da falta de consenso sobre a forma mais adequada para medir o grau de restrições financeiras, vários autores apontam este problema como uma barreira ao crescimento dos negócios, à inovação dos produtos e ao processo de internacionalização (e.g., Chaney, 2016), sendo as pequenas e médias empresas (PME) as mais afetadas (Berger \& Udell, 1998). No caso de Portugal, o tecido empresarial é formado maioritariamente por PME. De acordo com os dados do Instituto Nacional de Estatística (2019), as micro e PME ultrapassam na última década, em média, os $99 \%$ do total das empresas em Portugal. Neste contexto, o nosso objetivo principal é analisar o impacto das restrições financeiras no investimento e nas reservas de caixa das PME portuguesas do setor da metalomecânica. Este tópico tem sido deficientemente explorado, sendo que se destacam os estudos de Baptista, Matias e Valle (2013a, 2013b) para a indústria transformadora portuguesa. De acordo com este propósito, foi recolhida informação para empresas do setor da metalomecânica da base de dados SABI, entre 2006 e 2016. Em consonância com autores anteriores (e.g., Silva \& Carreira, 2011), as nossas hipóteses foram testadas através de modelos de efeitos fixos. Seguindo os estudos revistos (e.g., Gilchrist \& Himmelberg, 1995; Almeida, Campello \& Weisbach, 2004), a amostra foi dividida em empresas restritas e não restritas financeiramente de acordo com a dimensão e com as necessidades de financiamento. Os nossos resultados demonstram evidência de que as empresas financeiramente mais restritas revelam maior sensibilidade do investimento e das reservas de caixa relativamente aos fundos gerados internamente, medidos através da variável cash flow, o que corrobora o documentado por vários estudos revistos (e.g., Almeida et al., 2004; Fazzari et al., 1988).

Outro grande desafio colocado às PME é o da internacionalização. As empresas portuguesas têm procurado novos mercados como forma de ultrapassar as suas restrições financeiras. Segundo Silva (2011), a internacionalização das empresas pode melhorar o seu acesso ao financiamento externo. Consistente com este argumento, Silva e Carreira (2011) encontraram evidências de que as empresas exportadoras são menos dependentes de cash flow para investir do que as empresas domésticas. Neste sentido, também analisamos se as empresas domésticas do setor da metalomecânica dependem mais de financiamento gerado internamente para investir ou se mantêm maiores reservas de caixa do que as empresas exportadoras. Adicionalmente, estendemos esta hipótese e testamos se as empresas que exportam para o Mercado Comunitário são mais dependentes de financiamento gerado internamente do que as que exportam para dentro e fora do Mercado Comunitário, isto é, as que também exportam para o Mercado Extracomunitário. No entanto, e ao contrário da evidência anteriormente demonstrada na literatura, os nossos resultados sugerem que as empresas exportadoras apresentam maior sensibilidade do seu investimento e das reservas de caixa ao cash flow do que as empresas domésticas. E também no caso de as empresas que exportam para o Mercado Comunitário e Extracomunitário, estas aparentam ter maior sensibilidade do seu investimento e das reservas de caixa ao cash flow. 
Este trabalho está organizado da seguinte forma: a secção dois apresenta uma breve revisão de literatura relacionada com as hipóteses a testar; a secção três descreve a metodologia aplicada para testar as hipóteses de investigação, a amostra e as variáveis utilizadas; a secção quatro reporta e analisa os resultados empíricos. Por fim, na última secção são apresentadas as principais conclusões.

\section{Enquadramento teórico e formulação das hipóteses}

A literatura não é consensual quanto à melhor forma de medir as restrições financeiras sentidas pelas empresas no acesso ao financiamento externo. Neste sentido, Fazzari et al. (1988) propõem como proxy para capturar o grau de restrições financeiras o rácio de distribuição de dividendos. Partindo da análise a uma amostra de empresas cotadas norteamericanas, os autores concluem que quanto menor o rácio de distribuição de dividendos maior a sensibilidade do investimento ao cash flow. Desta forma, o rácio de distribuição de dividendos pode ser considerado um indicador do grau de racionamento financeiro enfrentado pelas empresas. Os autores encontram uma relação positiva e significativa entre o investimento e o cash flow, controlando as oportunidades de crescimento e dimensão. $O$ estudo seminal de Fazzari et al. (1988) foi, desde então, corroborado por outros estudos, que demonstraram evidência de suporte à hipótese da sensibilidade do investimento ao cash flow constituir um indicador adequado para mensurar as restrições financeiras enfrentadas pelas empresas, embora utilizando diferentes proxies para capturar o grau dessas restrições (e.g., Bond, Elston, Mairesse \& Mulkay, 2003; Bond \& Meghir, 1994; Calomiris \& Hubbard, 1990; Gilchrist \& Himmelberg, 1995; Hubbard, Kashyap \& Whited, 1995; Islam \& Mozumdar, 2007; Love, 2003).

Do outro lado desta discussão, Kaplan e Zingales (1997) analisaram o grupo de empresas consideradas como financeiramente restritas da amostra usada por Fazzari et al. (1988) e argumentam que os resultados encontrados pelos autores podem indicar apenas que as empresas não distribuem dividendos porque apresentam maior aversão ao risco, preferindo, assim, aumentar as reservas de caixa em vez de distribuir dividendos. Ao contrário de Fazzari et al. (1988), os resultados obtidos por Kaplan e Zingales (1997) evidenciam que as empresas consideradas como não restritas financeiramente apresentam maior sensibilidade do seu investimento ao cash flow. Estes resultados foram contrariados por Fazzari, Hubbard e Petersen (2000), que argumentam que Kaplan e Zingales (1997) classificam as empresas como financeiramente restritas as que apresentam um estado de asfixia financeira e, nesse caso, essas empresas vão utilizar os seus cash flows para fazer face às dívidas de curto prazo.

Mais tarde, Almeida et al. (2004) demonstram evidência de que as empresas que se deparam com maiores restrições financeiras tendem a conservar maiores reservas de caixa para minimizarem a possibilidade de perda de oportunidades de investimento derivada do risco da volatilidade dos seus cash flows futuros.

Com base nas evidências anteriores, formulamos a nossa primeira hipótese dividindo-a em duas alíneas: 
Hipótese 1.a: $O$ investimento das empresas consideradas financeiramente restritas apresenta maior sensibilidade às variações do cash flow.

Hipótese 1.b: As reservas de caixa das empresas consideradas financeiramente restritas apresentam maior sensibilidade às variações do cash flow.

A maioria dos estudos anteriores aponta as restrições financeiras como um bloqueio ao crescimento e à internacionalização das empresas (e.g., Caggese \& Cuñat, 2013; Chaney, 2016; Muûls, 2008). Consistente com esse argumento, Chaney (2016) aponta que, como os custos fixos associados à entrada em mercados externos são elevados, então, e em caso de as empresas serem restritas financeiramente, só empresas com suficiente liquidez inicial serão capazes de se internacionalizar. Este argumento corrobora as conclusões de vários autores (e.g., Bernard \& Jensen, 1999; Clerides, Lach \& Tybout, 1998; Ruano, Delgado \& Farinas, 2002) que demonstram que só as empresas mais produtivas e de maior dimensão terão capacidade de ser tornarem exportadoras, o que facilita o acesso a fundos externos.

Neste contexto, vários estudos empíricos analisaram o impacto das restrições financeiras na atividade exportadora das empresas. Musso e Schiavo (2008), com base numa amostra de empresas francesas, concluem que as empresas que se internacionalizam melhoram o seu desempenho financeiro. Também baseado numa amostra de empresas francesas, Bellone, Musso, Nesta e Schiavo (2010) concluem que as restrições financeiras formam uma barreira ao início da atividade exportadora porque a existência de custos elevados, para entrar em mercados externos, resultam em gastos superiores aos correspondentes rendimentos no início do processo.

Relativamente a evidências produzidas para o mercado português, Silva (2011) conclui para uma amostra de empresas da indústria transformadora que as restrições financeiras são um entrave às exportações e ao início da atividade exportadora. Neste contexto, Silva e Carreira (2011) analisaram o impacto das restrições financeiras numa amostra de PME e documentam que as empresas exportadoras têm mais facilidade de acesso a financiamento externo, pelo que enfrentam menos restrições financeiras.

Em consonância com os estudos anteriormente citados, formulamos as seguintes hipóteses:

Hipótese 2.a: O investimento das empresas domésticas é mais sensível ao cash flow do que o investimento das empresas exportadoras.

Hipótese 2.b: As reservas de caixa das empresas domésticas são mais sensíveis ao cash flow do que as reservas de caixa das empresas exportadoras.

Tal como apontado por diferentes autores (e.g., Chaney, 2016), as empresas podem ter que enfrentar custos quando decidem exportar para mercados internacionais, alguns destes custos são irrecuperáveis, outros associados a vários riscos, como risco político, risco país, risco comercial, entre outros. Mas se por um lado as empresas enfrentam barreiras à entrada dos mercados internacionais, por outro lado a atividade exportadora diversifica o risco e diminui os custos de financiamento (e.g., Silva, 2011). E, segundo a informação divulgada pelo Instituto Nacional de Estatística (2017), a maioria das empresas exportadoras portuguesas 
exporta, principalmente, para os Estados-membros da União Europeia (UE). No entanto, e dado o risco sistémico das economias da UE, principalmente as que se situam na Zona Euro, exportar para países fora da UE mitiga potencialmente o risco da atividade de exportação.

Neste contexto, a nossa última dupla de hipóteses postula o seguinte:

Hipótese 3.a: $O$ investimento das empresas que exportam somente para o Mercado Comunitário apresenta maior sensibilidade às variações do cash flow do que o investimento das empresas que exportam para o Mercado Comunitário e Extracomunitário.

Hipótese 3.b: As reservas de caixa das empresas que exportam somente para o Mercado Comunitário apresentam maior sensibilidade às variações do cash flow do que o investimento das empresas que exportam para o Mercado Comunitário e Extracomunitário.

\section{Descrição da amostra, variáveis e metodologia}

\subsection{Amostra}

De acordo com o objetivo deste trabalho, foi recolhida informação na base de dados SABI, entre 2006 e 2016, para as empresas pertencentes ao setor da metalomecânica (CAE 25). ${ }^{1}$

Os dados recolhidos contêm informações financeiras de PME pertencentes ao setor da metalomecânica, com um número mínimo de 10 e máximo de 250 funcionários, e volume de negócios de valor igual ou superior a dois milhões e máximo de cinquenta milhões de euros, de acordo com a definição de PME da diretiva europeia de 2003/361/CE.

Após a recolha dos dados foram aplicados vários filtros, eliminando dessa forma observações incorretas ou anómalas. ${ }^{2}$ De seguida, todas as variáveis sofreram um processo de winsorizing ${ }^{3}$ (e.g., Wilcox, 2012) ao nível de $1 \%$ em cada uma das caudas da distribuição, com o intuito de eliminar potenciais outliers que pudessem enviesar os resultados, invalidando, dessa forma, a inferência estatística.

Da aplicação destes filtros resultou uma amostra final com 12.771 observações respeitantes a 1.414 empresas.

\subsection{Definição das variáveis}

De acordo com o objetivo deste trabalho, e de forma a testarmos as hipóteses formuladas na secção anterior, selecionamos as seguintes variáveis baseadas nos estudos revistos (e.g., Almeida et al., 2004; Fazzari et al., 1988):

\section{Variáveis Dependentes}

\section{- Proxies de investimento:}

\footnotetext{
${ }^{1} \mathrm{O}$ CAE 25 diz respeito à fabricação de produtos metálicos, exceto máquinas e equipamentos.

${ }^{2}$ Observações com valores negativos nas seguintes variáveis: total do ativo, ativo corrente, ativo não corrente, volume de negócios, passivo não corrente, passivo corrente, total do passivo, depreciações e amortizações.

3 O winsorizing consiste em alterar os valores dos outliers para o valor da observação considerada não outlier de acordo com o nível proposto.
} 
- Capex (capital expenditures), rácio entre a variação absoluta do investimento em ativo fixo entre dois períodos consecutivos e o ativo total medido no início do período;

- Investimento medido como a variação percentual do ativo total entre dois períodos consecutivos. Esta proxy de investimento será usada como medida alternativa ao Capex.

- Reservas de Caixa, rácio entre a rubrica caixa e equivalentes e o ativo total medido no início do período.

\section{Variáveis Independentes}

- Cash flow medido como a soma do resultado líquido e das depreciações/amortizações, dividido pelo ativo total no início do período.

\section{Variáveis de Controlo}

- Dimensão medida através do logaritmo do ativo total.

- Oportunidade de crescimento medida como a variação percentual do volume de negócios entre dois períodos consecutivos. De acordo com diversos autores (e.g., Loureiro \& Silva, 2015), esta variável funciona como uma proxy que captura as oportunidades de crescimento.

As seguintes variáveis serão usadas somente na análise descritiva da amostra:

- Endividamento, rácio entre o passivo total e o ativo total.

- ROA (return on assets), rácio entre o EBIT (Earnings Before Interest and Taxes) e o total do ativo.

\section{Proxies das Restrições Financeiras}

- Dimensão medida da forma acima descrita. A Dimensão tem sido utilizada por vários autores como uma proxy das restrições financeiras (e.g., Gilchrist \& Himmelberg, 1995; Almeida et al., 2004), baseado no argumento de que empresas de maior dimensão enfrentam menores restrições financeiras;

- Necessidade de financiamento medida pela seguinte fórmula: ((Dividendos + Capex + Variações nas necessidades de fundo de maneio) - Cash Flow) / Total de ativos no início do período. Esta fórmula é uma adaptação do modelo de défice de financiamento proposto por Frank e Goyal (2003). As empresas que apresentem maiores necessidades de financiamento, e que não consigam colmatar essas necessidades com financiamento externo, demonstrarão maior sensibilidade do seu investimento (reservas de caixa) ao cash flow;

- Mercado Doméstico/Externo. São consideradas como exportadoras as empresas cujo volume de negócios para o mercado externo seja superior a 10\% do volume de negócios total e mantido em, pelo menos, dois anos durante o período da amostra. Todas as restantes observações que não cumpram esses critérios são consideradas domésticas; 
- Mercado Comunitário/Extracomunitário. As empresas exportadoras para o Mercado Comunitário mantêm relações comerciais apenas com Estados-membros da UE, enquanto as empresas exportadoras para o Mercado Comunitário e Mercado Extracomunitário também mantêm relações comerciais com países fora da UE.

A Tabela 1 apresenta as principais estatísticas descritivas das variáveis anteriormente descritas, respeitantes à amostra final, para o período 2006-2016.

Tabela 1. Resumo das estatísticas descritivas

\begin{tabular}{lrrrrrr}
\hline \multicolumn{1}{c}{ Variáveis } & N & Média & $\begin{array}{r}\text { Desvio } \\
\text { Padrão }\end{array}$ & Min & Mediana & Max \\
\hline Capex & 11357 & 0,0704 & 0,1179 & $-0,0761$ & 0,0285 & 0,6814 \\
Investimento & 11357 & 0,0550 & 0,1978 & $-0,4986$ & 0,0320 & 0,7387 \\
Reservas de Caixa & 11357 & 0,1123 & 0,1414 & 0,0003 & 0,0542 & 0,6774 \\
Cash Flow (CF) & 11357 & 0,0867 & 0,0874 & $-0,1729$ & 0,0725 & 0,3998 \\
Endividamento & 12771 & 0,6276 & 0,2252 & 0,1035 & 0,6531 & 1,3144 \\
ROA & 12771 & 0,0538 & 0,0758 & $-0,2156$ & 0,0419 & 0,3062 \\
Dimensão & 12771 & 7,3409 & 1,1127 & 5,0411 & 7,2204 & 10,0957 \\
Oportunidades de Crescimento & 11357 & 0,0660 & 0,2779 & $-0,5082$ & 0,0341 & 1,2479 \\
\hline A Tabela
\end{tabular}

A Tabela 1 apresenta as principais estatísticas descritivas da amostra para o período 2006-2016. As variáveis são as seguintes: Capex $=($ Ativo fixo[n] - Ativo fixo[n-1] + depreciações[n] $) /$ Ativo fixo[n-1]; Investimento=Total do ativo[n]/Total do ativo[n-1] - 1; Reservas de Caixa= Caixa e equivalentes[n]/Total do ativo[n-1]; $\mathrm{CF}=($ Resultados líquidos[n]+Dep./Amort.[n])/Total do ativo[n-1]; Endividamento = Passivo total/ Total do ativo; ROA = EBIT/Total do ativo; Dimensão = $\ln$ (Total do ativo); Oportunidades de Crescimento=Volume de Negócios[n]/Volume de Negócios[n-1] - 1. n é o número de observações.

Na Tabela 1, que descreve a amostra final, a primeira variável proxy do investimento (Capex) é, em média, de 7,04\%, enquanto o investimento apresenta um valor médio de 5,5\%. A variável Reservas de Caixa apresenta um peso médio de 11,23\% sobre o total de ativos, e o cash flow representa $8,67 \%$, em média, do ativo total. O rácio de endividamento corresponde, em média, a $62,76 \%$ do ativo total, o que é um sinal de que as empresas deste setor estão bastante alavancadas. A rendibilidade operacional do ativo (ROA) apresenta um valor médio de 5,38\%. A variável Oportunidades de Crescimento, baseada na taxa de crescimento das vendas, é positiva, registando um valor médio de $6,6 \%$ ao longo do período amostral.

A Tabela 2 apresenta a matriz de correlação de Pearson, que mede o grau de correlação linear entre as variáveis incluídas neste estudo. Podemos observar que os coeficientes de correlação são quase todos estatisticamente significativos ao nível de $1 \%$. De acordo com o esperado, os coeficientes das variáveis de investimento (Capex e Investimento) e a variável Reservas de Caixa estão positivamente correlacionados com o Cash flow e com a variável Oportunidades de Crescimento. Por sua vez, e também como esperado, o Endividamento está positivamente correlacionado as variáveis de investimento, o que sugere que estas empresas (também) recorrem a financiamento externo para suportarem os custos do investimento. Por outro lado, o Endividamento está negativamente correlacionado com o 
Cash flow e com as Reservas de Caixa, o que é justificado pelo facto de o Endividamento e o Cash flow serem fontes de financiamento concorrentes.

Relativamente à Dimensão, esta está correlacionada positivamente com as medidas de investimento, mas negativamente com a variável Reservas de Caixa. Isto significa que é esperado que as maiores empresas do setor da metalomecânica invistam mais em ativo fixo e que mantenham menores reservas de caixa do que as empresas de menor dimensão.

A análise de correlação é uma análise que tem a capacidade de sinalizar o movimento simultâneo de duas variáveis. Contudo, não distingue as causas dos seus efeitos, pelo que essa questão será tratada na próxima secção que descreve a metodologia.

Tabela 2. Matriz de correlação entre as variáveis

\begin{tabular}{|c|c|c|c|c|c|c|c|c|}
\hline & (1) & (2) & (3) & (4) & (5) & (6) & (7) & (8) \\
\hline Capex (1) & 1 & & & & & & & \\
\hline$p$-value & - & & & & & & & \\
\hline$N$ & 11357 & & & & & & & \\
\hline Investimento (2) & $0.5537^{*}$ & 1 & & & & & & \\
\hline$p$-value & 0,0000 & & & & & & & \\
\hline$N$ & 11357 & 11357 & & & & & & \\
\hline Reservas de Caixa (3) & $0.0442^{*}$ & $0.2271^{*}$ & 1 & & & & & \\
\hline$p$-value & 0,0000 & 0,0000 & & & & & & \\
\hline$N$ & 11357 & 11357 & 11357 & & & & & \\
\hline CF (4) & $0.3505^{*}$ & $0.4453^{*}$ & $0.3223^{*}$ & 1 & & & & \\
\hline$p$-value & 0,0000 & 0,0000 & 0,0000 & & & & & \\
\hline$N$ & 11357 & 11357 & 11357 & 11357 & & & & \\
\hline Endividamento (5) & $0.0842 *$ & $0.0745^{*}$ & $-0.3558^{*}$ & $-0.2461^{*}$ & 1 & & & \\
\hline$p$-value & 0,0000 & 0,0000 & 0,0000 & 0,0000 & & & & \\
\hline$N$ & 11357 & 11357 & 11357 & 11357 & 12771 & & & \\
\hline $\operatorname{ROA}(6)$ & $0.1070^{*}$ & $0.2740^{*}$ & $0.3079^{*}$ & $0.8467^{*}$ & $-0.2859^{*}$ & 1 & & \\
\hline$p$-value & 0,0000 & 0,0000 & 0,0000 & 0,0000 & 0,0000 & & & \\
\hline$N$ & 11357 & 11357 & 11357 & 11357 & 12771 & 12771 & & \\
\hline Dimensão (7) & $0.0479^{*}$ & $0.0895^{*}$ & $-0.1104^{*}$ & $0.0340^{*}$ & $-0.1048^{*}$ & $0.0208^{*}$ & 1 & \\
\hline$p$-value & 0,0000 & 0,0000 & 0,0000 & 0,0000 & 0,0000 & 0.0189 & & \\
\hline$N$ & 11357 & 11357 & 11357 & 11357 & 12771 & 12771 & 12771 & \\
\hline Oportunidades de Crescimento (8) & $0.2114 *$ & $0.3810^{*}$ & $0.0841^{*}$ & $0.3216^{*}$ & $0.0984^{*}$ & $0.2640^{*}$ & 0.0108 & 1 \\
\hline$p$-value & 0,0000 & 0,0000 & 0,0000 & 0,0000 & 0,0000 & 0,0000 & 0.2498 & - \\
\hline$N$ & 11357 & 11357 & 11357 & 11357 & 11357 & 11357 & 11357 & 11357 \\
\hline
\end{tabular}

A Tabela 2 apresenta os coeficientes de correlação de Pearson para a amostra final, recolhida para 0 período 2006-2016. As variáveis são as seguintes: Capex = (Ativo fixo[n] - Ativo fixo[n-1] + depreciações[n])/ Ativo fixo[n-1]; Investimento = Total do ativo[n]/Total do ativo[n-1] - 1; Reservas de Caixa = Caixa e equivalentes[n]/Total do ativo[n-1]; CF=(Resultados líquidos[n]+Dep./Amort.[n])/Total do ativo[n-1]; Endividamento = Passivo total $/$ Total do ativo; ROA = EBIT/Total do ativo; Dimensão = In(Total do ativo); Oportunidades de Crescimento =Volume de Negócios[n]/Volume de Negócios[n-1] - 1. * Significância estatística ao nível de, pelo menos, 5\%. O P-value e o número de observações reportados após a estimativa de cada coeficiente., respetivamente.

\subsection{Metodologia}

A nossa amostra final é um painel longitudinal de dados. Devido à heterogeneidade da amostra utilizada (transversal a diferentes empresas, localidades e anos), é esperado que os 
efeitos individuais não observáveis sejam constantes ao longo do tempo, sendo assim considerados como efeitos fixos (EF). No entanto, é de salientar que a escolha da metodologia no tratamento dos efeitos provocados pela heterogeneidade da amostra depende da aplicação de testes estatísticos adequados, como é o caso do teste de Hausman (1978). Este teste é baseado na existência, ou não, de correlação entre os efeitos individuais (não observáveis) e os regressores, sob a hipótese nula da não existência de correlação (sendo que nesse caso os efeitos são aleatórios).

A maioria dos estudos empíricos que usam dados em painel aplica a metodologia de EF devido à estrutura e natureza das suas amostras. A metodologia de EF permite controlar a heterogeneidade não observável entre as observações, confinando-a ao termo de interseção, e ao mitigar esse problema consegue-se uma melhoria na qualidade da estimação dos parâmetros. No entanto, os dados em painel podem ser afetados por problemas que enviesam a inferência estatística, tais como a heteroscedasticidade e a autocorrelação. De acordo com Cameron e Trivedi (2009), o método de clustering permite mitigar esses dois potenciais problemas ao estimar erros-padrão robustos baseando-se no pressuposto de que as observações, para a mesma empresa, estão correlacionadas em períodos temporais diferentes, mas as observações para as diferentes empresas não estão correlacionadas entre si.

Em consonância com as evidências demonstradas por Fazzari et al. (1988), a hipótese 1.a postula que o investimento está positivamente correlacionado com o cash flow, sendo que a magnitude dessa sensibilidade é maior para as empresas que apresentam maiores restrições financeiras. Desta forma, e de modo a testar essa hipótese, foram modeladas as seguintes equações:

$$
\begin{aligned}
& \text { Capex }_{i, t}=\beta_{0}+\beta_{1} C_{i, t}+\beta_{2} \Delta \text { Crescimento }_{i, t}+\beta_{3} \text { Dimensão }_{i, t}+\mu_{i}+\gamma_{t}+\varepsilon_{i, t} \\
& \text { Investimento }_{i, t}=\beta_{0}+\beta_{1} C_{i, t}+\beta_{2} \Delta \text { Crescimento }_{i, t}+\beta_{3} \text { Dimensão }_{i, t}+\mu_{i}+\gamma_{t}+\varepsilon_{i, t}
\end{aligned}
$$

Nesta equação, as variáveis dependentes são o Capex e o Investimento que são as proxies do investimento efetuado pelas empresas. A variável explicativa é o cash flow. Com a finalidade de controlar potenciais oportunidades de crescimento, foi incluída a variável Oportunidades de Crescimento ( $\Delta$ Crescimento), medida como a variação do volume de negócios. O logaritmo do total do ativo foi incluído para controlar a dimensão da empresa (Dimensão). A subsecção 3.2 descreve detalhadamente as variáveis. O termo $\mu_{\mathrm{i}}$ captura a heterogeneidade não observável. O parâmetro $\gamma_{t}$ é o conjunto de dummies que controla para os efeitos do tempo: $\varepsilon_{i, t}$ é o termo de erro.

De acordo com o postulado na hipótese 1.b, adaptamos o modelo de Almeida et al. (2004) e construímos a equação (3) para testarmos se as reservas de caixa estão positivamente correlacionadas com o cash flow e se essa sensibilidade é de maior magnitude nas empresas mais restritas financeiramente.

$$
\text { Reservas de Caixa }_{i, t}=\beta_{0}+\beta_{1} C_{i, t}+\beta_{2} \Delta \text { Crescimento }_{i, t}+\beta_{3} \text { Dimensão }_{i, t}+\mu_{i}+\gamma_{t}+\varepsilon_{i, t}
$$


Nesta formulação, a variável dependente corresponde às Reservas de Caixa. Todas as outras variáveis e termos são conforme descrito nas equações (1) e (2).

Numa primeira fase, foi aplicado às equações (1)-(3) o teste de Hausman (1978). De acordo com o esperado, os coeficientes do teste de Hausman conduzem à rejeição da hipótese nula de que os efeitos não observáveis são aleatórios, sendo que esses efeitos devem ser tratados como fixos. Estas evidências confirmam as suspeitas levantadas anteriormente, razão pela qual as equações de (1) a (3) foram modeladas para serem testadas através da metodologia de efeitos fixos.

Com o objetivo de testarmos as hipóteses 1.a e 1.b, a amostra final foi desagregada de acordo com o grau de restrições financeiras enfrentadas pelas empresas. Desta forma, a amostra foi dividida em empresas consideradas restritas financeiramente e empresas consideradas como não restritas, de acordo com duas proxies apontadas pelos estudos revistos pela sua capacidade de capturarem o grau das restrições financeiras: a Dimensão e as Necessidades de Financiamento. (e.g., Almeida et al., 2004; Frank \& Goyal, 2003). As empresas são classificadas como restritas ou não restritas financeiramente conforme cada observação se encontre abaixo ou acima da mediana, respetivamente, da variável Dimensão. No caso das Necessidades de Financiamento, as empresas são classificadas como restritas ou não restritas financeiramente conforme cada observação se encontre acima ou abaixo da mediana, respetivamente.

A divisão da amostra tendo em consideração a mediana é uma tendência da literatura sobre restrições financeiras (e.g., Gilchrist \& Himmelberg, 1995; Lins, Strickland \& Zenner, 2005). No que respeita aos critérios de classificação das empresas como exportadoras, e de acordo com Silva (2011), foram definidos como sendo 10\% do volume de negócios total verificado em, pelo menos, dois anos durante o período a que reporta a amostra.

\section{Análise empírica}

\subsection{Análise da sensibilidade do investimento e das reservas de caixa ao cash flow}

Esta secção tem como objetivo testar as hipóteses 1.a e 1.b. Tal como explicado na secção anterior, a amostra final foi desagregada de acordo com o grau de restrições financeiras enfrentadas pelas empresas em função das duas proxies das restrições financeiras usadas neste estudo: a Dimensão e as Necessidades de Financiamento. (e.g., Almeida et al., 2004; Frank \& Goyal, 2003).

As equações (1) a (3) foram reestimadas através da metodologia de efeitos fixos para cada um dos grupos das empresas e de acordo com cada uma das proxies de restrições financeiras, cujos resultados são apresentados na Tabela 3 abaixo.

Os resultados da Tabela 3 demonstram evidência de uma relação positiva e estatisticamente significativa entre o investimento, independentemente da forma como é medido (Capex e Investimento) e o cash flow, assim como uma relação positiva entre a variável Reservas de Caixa e o cash flow. Estes resultados são consistentes e robustos para qualquer uma das proxies usadas para capturar o grau de restrições financeiras (Dimensão ou Necessidades de Financiamento). No entanto, a magnitude da sensibilidade das variáveis de investimento (e variável Reservas de Caixa) ao cash flow parece ser superior para o grupo 
de empresas consideradas como financeiramente restritas, o que é consistente com as evidências anteriores (e.g., Fazzari et al., 1988). Esta evidência suporta as hipóteses 1.a e 1.b, que postulam que as empresas que enfrentam mais restrições financeiras apresentam maior sensibilidade do seu investimento e das suas reservas de caixa ao cash flow, respetivamente. Para confirmar estas hipóteses, testamos as diferenças entre os coeficientes da variável cash flow $\left(\beta_{1} C F_{i, t}\right)$ através de um teste $Z$, sob a hipótese nula de que as estimativas dos coeficientes são iguais $\left(\beta C F_{R}=\beta C F_{N R}\right)$. Como é possível observar nos Painéis $A$ e $B$ da Tabela 3 , todas as diferenças são estatisticamente significativas ao nível de $1 \%$, sendo só de realçar que no Painel A, a magnitude do coeficiente da relação entre a variável Investimento e o cash flow é significativamente superior no grupo das empresas consideradas como não restritas relativamente às empresas restritas. Esta contradição entre estimativas pode estar relacionada com a limitação da Dimensão se revelar uma proxy adequada para as restrições financeiras, tal como sugerido pelos autores Chirinko (1997), Kaplan e Zingales (1997) e Gomes (2001).

A título de exemplo, no Painel $\mathrm{B}$, a estimativa do coeficiente $\beta_{1}\left(C F_{i, t}\right)$ para o grupo de empresas consideradas restritas é de 0,2997 , enquanto o mesmo coeficiente para o grupo de empresas não restritas é de 0,1101. Assim, os resultados sugerem que quando o cash flow varia 1 ponto percentual, o Capex varia, em média, 0,2997 pontos percentuais no grupo de empresas consideradas restritas, enquanto a variação média no grupo de empresas consideradas como não restritas é de 0,1101 pontos percentuais, ceteris paribus. A hipótese da igualdade entre os coeficientes foi rejeitada, pelo que se conclui que as estimativas dos coeficientes são estatisticamente diferentes.

Esta evidência é também extensível à magnitude da relação entre a variável Reservas de Caixa e o cash flow, que é significativamente superior nas empresas consideradas restritas, o que sugere que as empresas que enfrentam mais restrições financeiras irão conservar maiores reservas de caixa como forma de preservar o investimento atual e de precaver o investimento futuro (Almeida et al., 2004).

Em suma, os resultados apresentados na Tabela 3 demonstram evidência que suporta as nossas hipóteses 1.a e 1.b.

\subsection{Análise da sensibilidade do investimento e das reservas de caixa ao cash flow das empresas domésticas versus empresas exportadoras}

A hipótese 2.a (2.b) postula que as empresas domésticas revelam maior sensibilidade do investimento (reservas de caixa) ao cash flow do que as empresas exportadoras. Para testar esta hipótese identificaram-se como exportadoras as empresas cujo volume de negócios para o exterior seja superior a 10\% do volume de negócios total, e que esse critério seja verificado em, pelo menos, dois anos durante o período amostral. As empresas consideradas domésticas são as restantes. A Tabela 4 reporta os resultados estimados com recurso à metodologia de efeitos fixos. 
Tabela 3. Sensibilidade do investimento e das reservas de caixa ao cash flow em função do grau de restrições financeiras

Painel A - Resultados da análise das sensibilidades ao cash flow

\begin{tabular}{|c|c|c|c|c|c|c|c|}
\hline Proxy & \multicolumn{7}{|c|}{ Dimensão } \\
\hline Grau de Restrição & \multicolumn{2}{|c|}{$\mathrm{R}$} & NR & $\mathrm{R}$ & NR & $\mathrm{R}$ & NR \\
\hline Equação & \multicolumn{3}{|c|}{ (1) } & \multicolumn{2}{|c|}{$(2)$} & \multicolumn{2}{|c|}{ (3) } \\
\hline Var. Dependentes & & & & \multicolumn{2}{|c|}{ INVESTIMENTO } & \multicolumn{2}{|c|}{ RESERVAS DE CAIXA } \\
\hline $\mathrm{CF}$ & \multicolumn{2}{|c|}{$\begin{array}{c}0,2789 * * * \\
(8,03)\end{array}$} & $\begin{array}{c}0,2746 * * * \\
(7,89)\end{array}$ & $0,9249 * * *$ & $\begin{array}{c}1,0957^{* * *} \\
(18,78)\end{array}$ & $\begin{array}{c}0,3536 * * * \\
(11,40)\end{array}$ & $\begin{array}{c}0,2809 * * * \\
(9,39)\end{array}$ \\
\hline$\triangle$ CRESCIMENTO & \multicolumn{2}{|c|}{$0,0177^{* *}$} & $0,0291 * * *$ & $0,1165^{* * *}$ & $\begin{array}{c}0,1121 * * * \\
(7,05)\end{array}$ & $\begin{array}{c}0,0109 * * \\
(2,38)\end{array}$ & $\begin{array}{c}0,0215 * * * \\
(3,66)\end{array}$ \\
\hline DIMENSÃO & \multicolumn{2}{|c|}{$\begin{array}{c}0,1351^{* * *} \\
(10,23)\end{array}$} & $\begin{array}{c}0,0836 * * * \\
(5,48)\end{array}$ & $\begin{array}{c}0,3070^{* * *} \\
(18,04)\end{array}$ & $\begin{array}{c}0,3407^{* * *} \\
(13,90)\end{array}$ & $\begin{array}{c}0,0377^{* * *} \\
(4,07)\end{array}$ & $\begin{array}{c}0,0363^{* * *} \\
(3,16)\end{array}$ \\
\hline Constante & \multicolumn{2}{|c|}{$-0,9826 * * *$} & $\begin{array}{c}-0,5725^{* * *} \\
(-5,22)\end{array}$ & $\begin{array}{c}-2,3720 * * * \\
(-18,28)\end{array}$ & $\begin{array}{c}-2,5129 * * * \\
(-14,32)\end{array}$ & $\begin{array}{c}-0,1876 * * * \\
(-2,63)\end{array}$ & $\begin{array}{c}-0,1694 * * \\
(-2,05)\end{array}$ \\
\hline $\mathrm{Z}$ test & \multicolumn{3}{|c|}{62,77} & \multicolumn{2}{|c|}{28,31} & \multicolumn{2}{|c|}{66,08} \\
\hline$P$-value $\beta C F_{R}=\beta C F_{N R}$ & \multicolumn{3}{|c|}{0,0000} & \multicolumn{2}{|c|}{0,0000} & & \\
\hline Observações & & & 4.768 & 6.583 & 4.768 & 6.583 & 4.768 \\
\hline $\mathrm{R}^{2}$ & & & 0,122 & 0,297 & 0,46 & 0,102 & 0,101 \\
\hline & ainel $B-R e$ & $\operatorname{tad}$ & da anális & e das sensibili & dades ao cas & h flow & \\
\hline Proxy & & & & cessidades de Fir & nanciamento & & \\
\hline Grau de Restrição & $\mathrm{R}$ & & $R$ & $\mathrm{R}$ & NR & $\mathrm{R}$ & NR \\
\hline Equação & & & & (2) & & $(3$ & \\
\hline Var. Dependentes & & & & INVESTIME & NTO & RESERVAS & E CAIXA \\
\hline $\mathrm{CF}$ & $\begin{array}{c}0,2997^{* * *} \\
(8,14)\end{array}$ & & $\begin{array}{l}1 \% * * \\
54)\end{array}$ & $\begin{array}{c}1,0140 * * * \\
(19,73)\end{array}$ & $\begin{array}{c}0,8423^{* * *} \\
(15,51)\end{array}$ & $\begin{array}{c}0,3355^{* * *} \\
(11,67)\end{array}$ & $\begin{array}{c}0,3236 * * * \\
(9,72)\end{array}$ \\
\hline$\triangle$ CRESCIMENTO & $\begin{array}{c}0,0184^{* *} \\
(2,25)\end{array}$ & $\begin{array}{r}0,0 \\
(\end{array}$ & $\begin{array}{l}30 * * * \\
74)\end{array}$ & $\begin{array}{c}0,1321^{* * *} \\
(9,44)\end{array}$ & $\begin{array}{c}0,1327^{* * *} \\
(9,55)\end{array}$ & $\begin{array}{c}0,0260 * * * \\
(4,71)\end{array}$ & $\begin{array}{l}0.0071 \\
(1,38)\end{array}$ \\
\hline DIMENSÃO & $\begin{array}{c}0,0736 * * * \\
(8,34)\end{array}$ & & 78) & $\begin{array}{c}0,2167^{* * *} \\
(17,08)\end{array}$ & $\begin{array}{c}0,2040 * * * \\
(17,73)\end{array}$ & $\begin{array}{c}0,0317^{* * *} \\
(4,61)\end{array}$ & $\begin{array}{c}0,0339 * * * \\
(4,17)\end{array}$ \\
\hline Constante & $\begin{array}{c}-0,4857^{* * *} \\
(-7,35)\end{array}$ & $\begin{array}{r}-0,3 \\
(-\end{array}$ & $\begin{array}{l}2 * * * \\
08)\end{array}$ & $\begin{array}{c}-1,6788 * * * \\
(-17,52)\end{array}$ & $\begin{array}{c}-1,5384 * * * \\
(-17,98)\end{array}$ & $\begin{array}{c}-0,1459^{* * *} \\
(-2,80)\end{array}$ & $\begin{array}{c}-0,1487^{* *} \\
(-2,45)\end{array}$ \\
\hline$Z$ test & & & & 58,99 & & 2,6 & \\
\hline$\beta C F_{R}=\beta C F_{N R}$ & & & & 0,0000 & & $0,0 c$ & \\
\hline Observações & 5.494 & & 357 & 5.494 & 5.857 & 5.494 & 5.857 \\
\hline $\mathrm{R}^{2}$ & 0,103 & & 66 & 0,342 & 0,283 & 0,124 & 0,099 \\
\hline
\end{tabular}

A Tabela 3 apresenta as estimativas dos coeficientes de regressão das equações (1) a (3) usando a metodologia EF. R significa "Restritas" e NR significa “Não Restritas" em função das proxies Dimensão e Necessidades de Financiamento. *, **, *** indicam um nível de significância de 10, 5 e 1\%, respetivamente. As variáveis são as seguintes: Capex = (Ativo fixo[n] - Ativo fixo[n-1] + depreciações[n])/ Ativo fixo[n-1]; Investimento = Total do ativo[n]/Total do ativo[n-1] - 1; Reservas de Caixa = Caixa e equivalentes[n]/Total do ativo[n-1]; CF = (Resultados líquidos[n]+Dep./Amort.[n])/Total do ativo[n-1]; $\Delta$ Crescimento = Volume de Negócios[n]/Volume de Negócios[n-1] - 1; Dimensão = In(Total do ativo). Os coeficientes do teste $t$ estão entre parênteses. O teste $Z$ é conduzido sobre a hipótese nula da igualdade entre coeficientes. P-value do teste $Z$ reportado. $R^{2}$ da regressão é reportado. 
Tabela 4. Sensibilidade do investimento e das reservas de caixa ao cash flow das empresas domésticas versus empresas exportadoras

\begin{tabular}{|c|c|c|c|c|c|c|}
\hline Grupo & Domésticas & Exportadoras & Domésticas & Exportadoras & Domésticas & Exportadoras \\
\hline Equação & \multicolumn{2}{|c|}{$(1)$} & \multicolumn{2}{|c|}{$(2)$} & \multicolumn{2}{|c|}{ (3) } \\
\hline Var. Dependentes & \multicolumn{2}{|c|}{ CAPEX } & \multicolumn{2}{|c|}{ INVESTIMENTO } & \multicolumn{2}{|c|}{ RESERVAS DE CAIXA } \\
\hline CF & $\begin{array}{c}0,2892^{* * *} \\
(10,46)\end{array}$ & $\begin{array}{c}0,3206^{* * *} \\
(5,78)\end{array}$ & $\begin{array}{c}0,9466 * * * \\
(23,40)\end{array}$ & $\begin{array}{c}1,0001 * * * \\
(14,19)\end{array}$ & $\begin{array}{c}0,3196 * * * \\
(12,81)\end{array}$ & $\begin{array}{c}0,3814 * * * \\
(7,11)\end{array}$ \\
\hline$\triangle$ CRESCIMENTO & $\begin{array}{c}0,0284^{* * *} \\
(4,81)\end{array}$ & $\begin{array}{c}0,0290 * * * \\
(2,60)\end{array}$ & $\begin{array}{c}0,1344 * * * \\
(11,63)\end{array}$ & $\begin{array}{c}0,1277^{* * *} \\
(5,95)\end{array}$ & $\begin{array}{c}0,0137^{* * *} \\
(3,33)\end{array}$ & $\begin{array}{c}0,0165^{* *} \\
(2,15)\end{array}$ \\
\hline DIMENSÃO & $\begin{array}{c}0,0633^{* * *} \\
(9,39)\end{array}$ & $\begin{array}{c}0,0691 * * * \\
(5,15)\end{array}$ & $\begin{array}{c}0,2153^{* * *} \\
(20,13)\end{array}$ & $\begin{array}{c}0,2067^{* * *} \\
(9,31)\end{array}$ & $\begin{array}{c}0,0343^{* * *} \\
(4,84)\end{array}$ & $\begin{array}{c}0,0122 \\
(1,15)\end{array}$ \\
\hline$Z$ test & \multicolumn{2}{|c|}{1,13} & \multicolumn{2}{|c|}{3,86} & \multicolumn{2}{|c|}{2,49} \\
\hline$\beta C F_{D}=\beta C F_{E X P}$ & \multicolumn{2}{|c|}{0,2585} & \multicolumn{2}{|c|}{0,0000} & \multicolumn{2}{|c|}{0,0128} \\
\hline Constante & $\begin{array}{c}-0,4328 * * * \\
(-8,56)\end{array}$ & $\begin{array}{c}-0,4604^{* * *} \\
(-4,77)\end{array}$ & $\begin{array}{c}-1,6636 * * * \\
(-20,62)\end{array}$ & $\begin{array}{c}-1,5464 * * * \\
(-9,45)\end{array}$ & $\begin{array}{c}-0,1618 * * * \\
(-3,00)\end{array}$ & $\begin{array}{l}0,0127 \\
(0,16)\end{array}$ \\
\hline Observações & 8.748 & 2.603 & 8.748 & 2.603 & 8.748 & 2.603 \\
\hline$R^{2}$ & 0,099 & 0,108 & 0,315 & 0,311 & 0,103 & 0,108 \\
\hline
\end{tabular}

A Tabela 4 apresenta as estimativas dos coeficientes de regressão para as equações (1) a (3) para duas subamostras: empresas domésticas versus empresas exportadoras. Os coeficientes das variáveis são estimados através da metodologia de EF. * **, *** indicam um nível de significância de 10,5 e $1 \%$, respetivamente. As variáveis são as seguintes: Capex = (Ativo fixo[n] - Ativo fixo[n-1] + depreciações[n])/ Ativo fixo[n-1]; Investimento = Total do ativo[n]/Total do ativo[n-1] - 1; Reservas de Caixa = Caixa e equivalentes[n]/Total do ativo[n-1]; CF = (Resultados líquidos[n]+Dep./Amort.[n])/Total do ativo[n-1]; $\Delta$ Crescimento $=$ Volume de Negócios[n]/Volume de Negócios[n-1] $-1 ;$ Dimensão $=$ In(Total do ativo). Os coeficientes do teste $t$ estão entre parênteses. São consideradas exportadoras as empresas cujo volume de negócios para o exterior seja superior a 10\% em, pelo menos, 2 anos do período amostral e as empresas consideradas domésticas são as que não cumprem estes critérios. $O$ teste $Z$ é conduzido sobre a hipótese nula da igualdade entre coeficientes. $P$-value do teste $Z$ reportado. $\mathrm{R}^{2}$ da regressão é reportado.

Os resultados da Tabela 4 demonstram evidência de uma relação positiva e significativa ao nível de $1 \%$ entre as variáveis de investimento (e a variável Reservas de Caixa) e o cash flow, tanto para o grupo de empresas consideradas domésticas como para as empresas consideradas exportadoras. No entanto, ao contrário do esperado e postulado na hipótese 2.a (2.b), a magnitude dos coeficientes que exprimem a sensibilidade do investimento (Reservas de Caixa) ao cash flow é superior para as empresas exportadoras. Contudo, a diferença entre esses coeficientes só é significativa no caso da variável Investimento e da variável Reservas de Caixa. Estes resultados são contrários ao formulado na hipótese 2, que postula que as empresas exportadoras são menos restritas financeiramente e, por isso, o seu investimento é menos sensível ao cash flow do que o investimento das empresas domésticas (e.g., Silva \& Carreira, 2011). O coeficiente da relação entre a variável Reservas de Caixa e o cash flow também apresenta maior magnitude para o grupo de empresas exportadoras, sendo esta evidência contrária aos resultados de Silva e Carreira (2011) que demonstram que 
as empresas domésticas revelam maior sensibilidade de Reservas de Caixa e equivalentes ao cash flow relativamente às empresas exportadoras.

A título de exemplo, quando o cash flow varia 1 ponto percentual, a variável Reservas de Caixa varia 0,3196 pontos percentuais para a média das empresas domésticas e 0,3814 pontos percentuais para a média das empresas exportadoras, ceteris paribus. Uma possível justificação para estes resultados contrários ao previsto são os elevados custos de entrada em mercado externos documentados pelos estudos revistos (e.g., Caggese \& Cuñat, 2013; Chaney, 2016; Muûls, 2008). Talvez as empresas consideradas como exportadoras na nossa amostra estejam numa fase de entrada em novos mercados, estando ainda a suportar esses custos. Diversos autores apontam para os benefícios da internacionalização no desempenho financeiro das empresas, ajudando-as a ultrapassar parte das suas restrições financeiras (e.g., Silva, 2011; Silva \& Carreira, 2011). No entanto, o processo de internacionalização pode impor custos avultados às empresas, principalmente, às empresas de menor dimensão (e.g. Chaney, 2016).

Em conclusão, os resultados da Tabela 4 não suportam a hipótese 2.

\subsection{Análise da sensibilidade do investimento e das reservas de caixa ao cash flow: Exportadoras para o mercado comunitário versus exportadoras para o mercado comunitário e extracomunitário}

De acordo com a hipótese 3, é esperado que o investimento e as reservas de caixa das empresas consideradas exportadoras que mantêm relações comerciais apenas com Estadosmembros da UE (isto é, dentro do Mercado Comunitário) sejam mais sensíveis ao cash flow do que o investimento e as reservas de caixa das empresas que exportam também para outros países fora do Mercado Comunitário.

Para testarmos essa hipótese, as equações (1) a (3) foram reestimadas com recurso à metodologia de efeitos fixos, para cada um dos grupos: i) grupo de empresas que exportam somente para o Mercado Comunitário e ii) grupo de empresas que exportam para o Mercado Comunitário e para o Mercado Extracomunitário. A Tabela 5 apresenta os resultados desta análise.

Tal como esperado, os resultados reportados na Tabela 5 demonstram evidência de uma relação positiva e estatisticamente significativa entre as variáveis de investimento (e a variável Reservas de (aixa) e o cash flow para os dois grupos: i) empresas que exportam somente para o Mercado Comunitário e ii) empresas que exportam para o Mercado Comunitário e Extracomunitário. O nível de significância de todos os coeficientes associados é de $1 \%$.

A hipótese 3.a (3.b) postula que as empresas que exportam para o Mercado Comunitário e Extracomunitário revelam menor sensibilidade do seu investimento (e variável Reservas de Caixa) ao cash flow dado sentirem menos restrições financeiras no acesso ao financiamento externo do que as empresas que exportam somente para o Mercado Comunitário, o que é consistente com a hipótese do efeito de diversificação; o aumento dos mercados externos com os quais se mantêm relações comerciais conduz a uma diminuição da taxa de custo do financiamento (e.g., Chaney, 2016). 
Tabela 5. Sensibilidade do investimento e das reservas de caixa ao cash flow: exportadoras para o mercado comunitário versus exportadoras para o mercado comunitário e extracomunitário

\begin{tabular}{|c|c|c|c|c|c|c|}
\hline Grupo & $\begin{array}{c}\text { Mercado } \\
\text { Comunitário }\end{array}$ & $\begin{array}{c}\text { Mercado } \\
\text { Extra } \\
\text { Comunitário }\end{array}$ & $\begin{array}{c}\text { Mercado } \\
\text { Comunitário }\end{array}$ & $\begin{array}{c}\text { Mercado } \\
\text { Extra } \\
\text { Comunitário }\end{array}$ & $\begin{array}{c}\text { Mercado } \\
\text { Comunitário }\end{array}$ & $\begin{array}{c}\text { Mercado } \\
\text { Extra } \\
\text { Comunitário }\end{array}$ \\
\hline Equação & \multicolumn{2}{|c|}{ (1) } & \multicolumn{2}{|c|}{ (2) } & \multicolumn{2}{|c|}{ (3) } \\
\hline Var. Dependentes & \multicolumn{2}{|c|}{ CAPEX } & \multicolumn{2}{|c|}{ INVESTIMENTO } & \multicolumn{2}{|c|}{ RESERVAS DE CAIXA } \\
\hline $\mathrm{CF}$ & $\begin{array}{c}0,2291 * * * \\
(6,22)\end{array}$ & $\begin{array}{c}0,3229 * * * \\
(8,11)\end{array}$ & $\begin{array}{c}0,8918 * * * \\
(16,65)\end{array}$ & $\begin{array}{c}1,0001 * * * \\
(15,94)\end{array}$ & $\begin{array}{c}0,3168 * * * \\
(9,67)\end{array}$ & $\begin{array}{c}0,3365^{* * *} \\
(8,85)\end{array}$ \\
\hline$\triangle$ CRESCIMENTO & $\begin{array}{c}0,0226 * * * \\
(2,94)\end{array}$ & $\begin{array}{c}0,0215^{* * *} \\
(2,73)\end{array}$ & $\begin{array}{c}0,1229 * * * \\
(8,19)\end{array}$ & $\begin{array}{c}0,1277^{* * *} \\
(7,44)\end{array}$ & $\begin{array}{c}0,0151^{* *} \\
(2,55)\end{array}$ & $\begin{array}{c}0,0176 * * * \\
(2,98)\end{array}$ \\
\hline DIMENSÃO & $\begin{array}{c}0,0817^{* * *} \\
(7,96)\end{array}$ & $\begin{array}{c}0,0770 * * * \\
(8,50)\end{array}$ & $\begin{array}{c}0,2669 * * * \\
(17,84)\end{array}$ & $\begin{array}{c}0,2397^{* * *} \\
(13,95)\end{array}$ & $\begin{array}{c}0,0317^{* * *} \\
(4,00)\end{array}$ & $\begin{array}{c}0,0291 * * * \\
(2,93)\end{array}$ \\
\hline Constante & $\begin{array}{c}-0,5556 * * * \\
(-7,39)\end{array}$ & $\begin{array}{c}-0,5381 * * * \\
(-7,96)\end{array}$ & $\begin{array}{c}-2,0212 * * * \\
(-18,24)\end{array}$ & $\begin{array}{c}-1,8446 * * * \\
(-14,18)\end{array}$ & $\begin{array}{c}-0,1366 * * \\
(-2,31)\end{array}$ & $\begin{array}{l}-0.1219 \\
(-1,63)\end{array}$ \\
\hline Z test & \multicolumn{2}{|c|}{31,78} & \multicolumn{2}{|c|}{11,79} & \multicolumn{2}{|c|}{3,77} \\
\hline$\beta C F_{\text {Intra }}=\beta C F_{\text {Extra }}$ & \multicolumn{2}{|c|}{0,0000} & \multicolumn{2}{|c|}{0,0000} & \multicolumn{2}{|c|}{0,0002} \\
\hline Observações & 4.926 & 4.681 & 4.926 & 4.681 & 4.926 & 4.681 \\
\hline $\mathrm{R}^{2}$ & 0,079 & 0,113 & 0,309 & 0,337 & 0,103 & 0,104 \\
\hline
\end{tabular}

A Tabela 5 apresenta as estimativas dos coeficientes de regressão para as equações (1) a (3) de acordo com os grupos em análise: empresas que exportam somente para o Mercado Comunitário e empresas que exportam para o Mercado Comunitário e Extracomunitário. Os coeficientes das variáveis são estimados através da metodologia de EF. *,**, *** indicam um nível de significância de 10, 5 e 1\%, respetivamente. As variáveis são as seguintes: Capex = (Ativo fixo[n] - Ativo fixo[n-1] + depreciações[n])/ Ativo fixo[n-1]; Investimento = Total do ativo[n]/Total do ativo[n-1] - 1; Reservas de Caixa = Caixa e equivalentes[n]/Total do ativo[n-1]; CF = (Resultados líquidos[n]+Dep./Amort.[n])/Total do ativo[n-1]; $\Delta$ Crescimento = Volume de Negócios[n]/Volume de Negócios[n-1] - 1; Dimensão = In(Total do ativo). Os coeficientes do teste $t$ estão entre parênteses. O teste $Z$ é conduzido sobre a hipótese nula da igualdade entre coeficientes. $P$-value do teste $Z$ reportado. $R^{2}$ da regressão é reportado.

No entanto, os nossos resultados demonstram evidência do contrário; as estimativas dos coeficientes da variável cash flow são de maior magnitude para o grupo que exporta também para o Mercado Extracomunitário (além de exportar para o Mercado Comunitário) e a diferença entre os coeficientes dos dois grupos é estatisticamente significativa (confirmado pelos coeficientes do teste $Z$ ). Ao contrário da evidência anteriormente demonstrada na literatura (Bridges \& Guariglia, 2008; Silva \& Carreira, 2011), os nossos resultados sugerem que as empresas exportadoras apresentam maior sensibilidade do seu investimento e das reservas de caixa ao cash flow do que as empresas domésticas, assim como as empresas que exportam para o Mercado Extracomunitário (além do Mercado Comunitário) aparentam ter maior sensibilidade do seu investimento e das reservas de caixa ao cash flow. Uma potencial justificação para os nossos resultados pode estar relacionada com o período temporal analisado. De acordo com os dados do Banco de Portugal (2015, 2017), o número de empresas do setor da Metalomecânica tem aumentado, o que significa empresas mais jovens que sofrem maiores barreiras à entrada dos mercados internacionais. Os dados fornecidos pela 
Central de Balanços do Banco de Portugal (2015, 2017) mostram que durante o período amostral (2006-2016), o contributo do mercado externo para o total do volume de negócios do setor da metalomecânica passa de negativo para positivo (-17 pontos percentuais em 2009 para 10 pontos percentuais em 2010). Este crescimento do volume de negócios para o mercado externo sugere avultados investimentos por parte das empresas para dar resposta à oportunidade de internacionalização, o que pode ter conduzido as empresas a recorrerem a fundos externos com a expetativa de aumentarem as receitas. Nos anos seguintes a 2010, este contributo das vendas para o exterior no volume de negócios desce, e apenas em 2013 a taxa de crescimento do volume de negócios volta a subir impulsionada pelas vendas para o mercado externo, mas a descida do volume de negócios nos anos anteriores a 2013 não foi compensada por uma subida da mesma magnitude nos anos subsequentes. Estes dados estatísticos sugerem que as receitas geradas nos anos seguintes ao avultado investimento inicial efetuado por parte das empresas para se internacionalizarem terão sido insuficientes para cobrirem os custos.

Em suma, os nossos resultados são contrários à evidência anterior (e.g., Caggese \& Cuñat, 2013; Chaney, 2016; Muûls, 2008) e podem estar associados aos custos de entrada em novos mercados. Outra explicação possível é um potencial erro de mensuração das oportunidades de crescimento; quando existe um erro de mensuração nessa variável, o cash flow pode constituir, em simultâneo, uma proxy para fundos gerados internamente e para as oportunidades de crescimento, o que pode ampliar a sua magnitude.

Em suma, a nossa evidência não suporta a hipótese 3.

\section{Conclusão}

A motivação do nosso estudo prende-se com a análise às restrições financeiras que afetam o acesso ao financiamento externo das empresas de pequena e média dimensão que dominam o tecido empresarial Português. Tanto quanto é de nosso conhecimento, este é o primeiro estudo a demonstrar evidência sobre a sensibilidade do investimento e das reservas de caixa ao financiamento interno para as empresas do setor da Metalomecânica em Portugal.

De acordo com o nosso objetivo, recolhemos uma amostra de PME portuguesas do setor da metalomecânica (CAE 25), entre 2006 e 2016. A nossa amostra final resultou em 12771 observações correspondentes a 1414 empresas.

Com base na evidência demonstrada nos estudos revistos, testámos as nossas hipóteses dividindo as empresas em restritas e não restritas financeiramente de acordo com duas proxies que captam essas restrições: a Dimensão e as Necessidades de Financiamento.

A nossa evidência é consistente com Fazzari et al. (1988); as empresas consideradas como mais restritas revelam maior sensibilidade do seu investimento ao cash flow. Em consonância com o estudo de Almeida et al. (2004), os nossos resultados sugerem que as empresas que enfrentam mais restrições financeiras irão conservar maiores reservas de caixa em função do cash flow. Em suma, os nossos resultados suportam as hipóteses 1.a e 1.b.

Neste sentido, também testámos se as empresas domésticas do setor da metalomecânica são mais dependentes de financiamento interno do que as empresas exportadoras. No entanto, e ao contrário do esperado e postulado na hipótese 2.a (2.b), a 
sensibilidade do investimento (Reservas de Caixa) ao cash flow é superior para as empresas exportadoras. Estes resultados são contrários aos apontados na literatura anterior, que defende que as empresas exportadoras são menos restritas financeiramente e, por isso, o seu investimento (reservas de caixa) é menos sensível ao cash flow do que o investimento (reservas de caixa) das empresas domésticas (e.g., Silva \& Carreira, 2011). Deste modo, os resultados não suportam a hipótese 2.a (2.b).

Seguindo a mesma intuição, estendemos esta análise às empresas que exportam para o Mercado Comunitário e as que exportam para o Mercado Comunitário e Extracomunitário. Mais uma vez, os nossos resultados são contrários à evidência anterior (e.g., Caggese \& Cuñat, 2013; Chaney, 2016; Muûls, 2008), pelo que as empresas que exportam para o Mercado Comunitário e Extracomunitário enfrentam mais restrições financeiras do que as empresas que exportam somente para o Mercado Comunitário. Deste modo, a nossa evidência não suporta a hipótese 3 .

Apesar da evidência demonstrada, é importante mencionar que este estudo está sujeito a limitações. A maior limitação é a falta de informação disponível para PME, sendo que a qualidade da informação disponível também é questionável dado que a maioria das PME não está sujeita a auditorias financeiras de acordo com o enquadramento legal em vigor. Essa falta de informação limita a construção de outras variáveis de controlo ou proxies que captem as restrições financeiras consideradas pelos estudos revistos como mais adequadas.

Esta limitação pode influenciar o impacto da internacionalização como fator mitigador das restrições financeiras.

Neste contexto, sugere-se como possível linha de investigação a elaboração de um inquérito dirigido aos diretores financeiros das empresas da nossa amostra sobre a sua perceção relativamente ao grau de restrições financeiras que enfrentam.

\section{Referências}

Almeida, H., Campello, M. \& Weisbach, M. S. (2004). The cash flow sensitivity of cash. The Journal of Finance, 59(4), 1777-1804. DOI: 10.1111/j.1540-6261.2004.00679.x

Banco de Portugal. (2015). Análise setorial da indústria metalomecânica 2009-2013. Disponível em https://www.bportugal.pt/

Banco de Portugal. (2017). Análise setorial da indústria metalomecânica 2011-2016. Disponível em https://www.bportugal.pt/

Baptista, C., Matias, F. \& Valle P. (2013a). Fatores moderadores da dependência do investimento relativamente à liquidez interna. Tourism \& Management Studies, 9(2), 71-77.

Baptista, C., Matias, F. \& Valle, P. (2013b). The moderating role of strategy and environment on the relationship between corporate liquidity and investment: Evidence from panel data. Tourism \& Management Studies, 9(1), 85-91.

Bellone, F., Musso, P., Nesta, L. \& Schiavo, S. (2010). Financial constraints and firm export behaviour. The World Economy, 33(3), 347-373. DOI: 10.1111/j.1467-9701.2010.01259.x

Berger, A. \& F. Udell, G. (1998). The economics of small business finance: The roles of private equity and debt markets in the financial growth cycle. Journal of Banking \& Finance, 22(6-8), 613-673. DOI: 10.1016/S0378-4266(98)00038-7 
Bernard, A. B. \& Jensen, J. B. (1999). Exceptional exporter performance: Cause, effect, or both? Journal of International Economics, 47, 1-25. DOI: 10.3386/w6272

Bond, S., Elston, J. A., Mairesse, J. \& Mulkay, B. (2003). Financial factors and investment in Belgium, France, Germany, and the United Kingdom: A comparison using company panel data. The Review of Economics and Statistics, 85(1), 153-165. DOI: 10.1162/003465303762687776

Bond, S. \& Meghir, C. (1994). Dynamic investment models and the firm's financial policy. Review of Economic Studies, 61(2), 197-222. DOI: 10.2307/2297978

Bridges, S. \& Guariglia, A. (2008). Financial constraints, global engagement, and firm survival in the UK: Evidence from micro data. Scottish Journal of Political Economy, 55(4), 444-464. DOI: 10.1111/j.1467-9485.2008.00461.x

Caggese, A. \& Cuñat, V. (2013). Financing constraints, firm dynamics, export decisions, and aggregate productivity. Review of Economic Dynamics, 16, 177-193. DOI: 10.1016/j.red.2012.10.004

Calomiris, C. W. \& Hubbard, R. G. (1990). Firm heterogeneity, internal finance, and credit rationing. The Economic Journal, 100(399), 90-104. DOI: 10.2307/2233596

Cameron, A. C. \& Trivedi, P. K. (2009). Microeconometrics Using Stata. Texas: Stata Press.

Chaney, T. (2016). Liquidity constrained exporters. Journal of Economic Dynamics \& Control, 72, 141-154. DOI: 10.1016/j.jedc.2016.03.010

Chirinko, R. (1997). Finance constraints, liquidity, and investment spending: Theoretical restrictions and international evidence. Journal of the Japanese and International Economies, 11(2), 185-207. DOI: $10.1006 /$ jjie.1997.0376

Clerides, S., Lach, S. \& Tybout, J. (1998). Is “Learning-by-Exporting” important? Micro-dynamic Evidence from Colombia, Mexico and Morocco. The Quarterly Journal of Economics, 113(3), 903-947.

Fazzari, S., Hubbard, G. \& Petersen, B. (1988). Financing constraints and corporate investment. Brookings Papers on Economic Activity, 19(1), 141-206. DOI: 10.2307/2534426

Fazzari, S., Hubbard, G. \& Petersen, B. (2000). Investment-cash flow sensitivities are useful: A comment on Kaplan and Zingales. The Quarterly Journal of Economics, 115(2), 695-705.

Frank, M. \& Goyal, V. (2003). Testing the pecking order theory of capital structure. Journal of Financial Economics, 67, 217-248. DOI: 10.1016/S0304-405X(02)00252-0

Gilchrist, S. \& Himmelberg, C. P. (1995). Evidence on the role of cash flow for investment. Journal of Monetary Economics, 36(3), 541-572. DOI: 10.1016/0304-3932(95)01223-0

Gomes, J. F. (2001). Financing investment. American Economic Review, 91(5), 1263-1285. DOI: 10.1257/aer.91.5.1263.

Hausman, J. A. (1978). Specification tests in econometrics. Econometrica, 46(6), 1251-1271. DOI: $10.2307 / 1913827$

Hubbard, R. G., Kashyap, A. K. \& Whited, T. M. (1995). Internal finance and firm investment. Journal of Money, Credit \& Banking, 27(3), 683-701. DOI: 10.2307/2077743

Instituto Nacional de Estatística (2017, 31 de julho). Perfil Exportador das Sociedades 2010-2015. Disponível em: https://www.ine.pt/xportal/xmain?xpid=INE\&xpgid=ine_destaques\&DESTAQUESdest_boui=29 9265718\&DESTAQUESmodo $=2$

Instituto Nacional de Estatística (2019). Estatísticas das Empresas 2017. Disponível em https://www.ine.pt/xportal/xmain?xpid=INE\&xpgid=ine_publicacoes\&PUBLICACOESpub_boui= 358541793\&PUBLICACOESmodo $=2$

Islam, S. S. \& Mozumdar, A. (2007). Financial market development and the importance of internal cash: Evidence from international data. Journal of Banking and Finance, 31(3), 641-658. DOI: 


\subsection{6/j.jbankfin.2006.06.009}

Kaplan, S. N. \& Zingales, L. (1997). Do investment-cash flow sensitivities provide useful measures of financing constraints? The Quarterly Journal of Economics, 112(1), 169-215.

Lins, K., Strickland, D. \& Zenner, M. (2005). Do non-U.S. firms issue equity on U.S. exchanges to relax capital constraints? Journal of Financial and Quantitative Analysis, 40(1), 109-133. DOI: 10.1017/S0022109000001769.

Loureiro, G. \& Silva, S. (2015). Cross-delisting, financial constraints and investment sensitivities. NIPE Working Papers Series. Universidade do Minho.

Love, I. (2003). Financial development and financing constraints: International evidence from the structural investment model. The Review of Financial Studies, 16(3), 765-791. DOI: $10.1093 / \mathrm{rfs} / \mathrm{hhg} 013$

Musso, P. \& Schiavo, S. (2008). The impact of financial constraints on firm survival and growth. Journal of Evolutionary Economics, 18(2), 135-149. DOI: 10.1007/s00191-007-0087-z

Muûls, M. (2008). Exporters and credit constraints. A firm-level approach. Working Paper Research 139. National Bank of Belgium.

Ruano, S., Delgado, M. A. \& Farinas, J. C. (2002). Firm productivity and export markets: A non-parametric approach. Journal of International Economics, 57(2), 397-422. DOI: 10.1016/S00221996(01)00154-4

Silva, A. (2011). Financial constraints and exports: Evidence from Portuguese manufacturing firms. International Journal of Economic Sciences and Applied Research Financial, 4(3), 7-19.

Silva, F. \& Carreira, C. (2011). Financial constraints and exports: An analysis of Portuguese firms during the European monetary integration. Notas Económicas, 34, 35-56.

Wilcox, R. (2012). Introduction to robust estimation and hypothesis testing. International Edition: Academic Press, Elsevier.

MARÍLIA VEIGA Mestre em Finanças Empresariais pelo Instituto Superior de Contabilidade e Administração do Porto (ISCAP) - Instituto Politécnico do Porto. Licenciada em Gestão de Empresas pelo Instituto Superior de Línguas e Administração (ISLA). Atualmente ocupa a posição de Assistente Técnica no Museu Nacional de Soares dos Reis. Endereço institucional: ISCAP, Instituto Politécnico do Porto. R. Jaime Lopes Amorim s/n, 4465-004 Matosinhos.

SÓNIA SILVA Investigadora doutorada no Núcleo de Investigação em Políticas Económicas da Escola de Economia e Gestão da Universidade do Minho. Doutorada em Ciências Empresariais (especialidade em Finanças Empresariais) pela Universidade do Minho. Foi Professora Convidada durante 10 anos no ensino superior em diferentes instituições (Universidade do Minho, Universidade de Aveiro, Instituto Superior de Administração e Gestão e Instituto Politécnico do Porto). Endereço institucional: Escola Economia e Gestão, Universidade do Minho, Campus de Gualtar, 4710-057 Braga.

Submetido em 11 janeiro 2019

Aceite em 26 abril 2019 